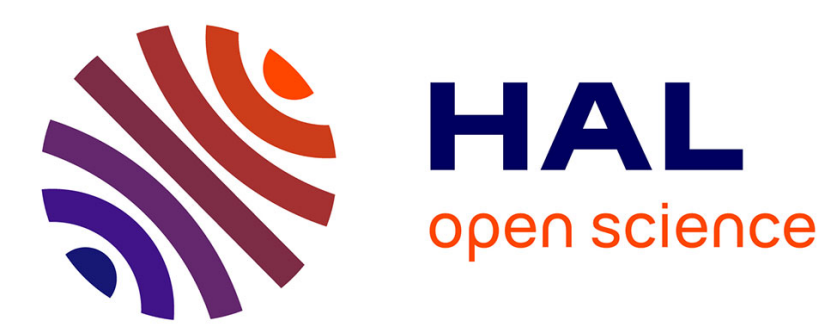

\title{
Aspects of lateral resolution in energy-filtered core level photoelectron emission microscopy
}

\author{
Aude Bailly, Olivier Renault, Nicholas Barrett, Tristan Desrues, Denis
}

Mariolle, Luiz Fernando Zagonel, Matthias Escher

\section{- To cite this version:}

Aude Bailly, Olivier Renault, Nicholas Barrett, Tristan Desrues, Denis Mariolle, et al.. Aspects of lateral resolution in energy-filtered core level photoelectron emission microscopy. Journal of Physics: Condensed Matter, 2009, 21, pp.314002. 10.1088/0953-8984/21/31/314002 . hal-00685501

\section{HAL Id: hal-00685501 https://hal.science/hal-00685501}

Submitted on 5 Apr 2012

HAL is a multi-disciplinary open access archive for the deposit and dissemination of scientific research documents, whether they are published or not. The documents may come from teaching and research institutions in France or abroad, or from public or private research centers.
L'archive ouverte pluridisciplinaire $\mathbf{H A L}$, est destinée au dépôt et à la diffusion de documents scientifiques de niveau recherche, publiés ou non, émanant des établissements d'enseignement et de recherche français ou étrangers, des laboratoires publics ou privés. 


\title{
Aspects of lateral resolution in energy-filtered core level photoelectron emission microscopy
}

\author{
A Bailly ${ }^{1}$, O Renault ${ }^{1}$, N Barrett ${ }^{2}$, T Desrues ${ }^{1,4}$, D Mariolle ${ }^{1}$, \\ L F Zagonel $^{2}$ and $M$ Escher $^{3}$ \\ ${ }^{1}$ CEA, LETI, MINATEC, 17 rue des Martyrs, 38054 Grenoble Cedex 9, France \\ ${ }^{2}$ CEA DSM/IRAMIS/SPCSI, CEA Saclay, 91191 Gif-sur-Yvette, France \\ ${ }^{3}$ FOCUS GmbH, Neukirchner Straße 2, 65510 Hünstetten-Kesselbach, Germany \\ E-mail: orenault@cea.fr
}

Received 18 December 2008, in final form 5 February 2009

Published 7 July 2009

Online at stacks.iop.org/JPhysCM/21/314002

\begin{abstract}
Lateral resolution is a major issue in photoelectron emission microscopy (PEEM) and received much attention in the past; however a reliable practical methodology allowing for inter-laboratory comparisons is still lacking. In modern, energy-filtered instruments, core level or valence electrons give much lower signal levels than secondary electrons used in still most of the present experiments. A quantitative measurement of the practical resolution obtained with core level electrons is needed. Here, we report on critical measurements of the practical lateral resolution measured for certified semiconducting test patterns using core level photoelectrons imaged with synchrotron radiation and an x-ray PEEM instrument with an aberration-corrected energy filter. The resolution is $250 \pm 20 \mathrm{~nm}$ and the sensitivity, $38 \mathrm{~nm}$. The different contributions to the effective lateral resolution (electron optics, sample surface imperfections, counting statistics) are presented and quantitatively discussed.
\end{abstract}

(Some figures in this article are in colour only in the electronic version)

\section{Introduction}

Lateral resolution is a major issue in photoelectron emission microscopy (PEEM) and received much attention in the past $[1,2]$. One of the major future directions of $\mathrm{x}$ ray PEEM (XPEEM) is energy filtering [3, 4] enabling core level spectromicroscopy at high lateral and energy resolution. Particularly important in energy-filtered XPEEM is the application to single nano-objects $[5,6]$ and nanopatterned structures with its ability to provide unique spectroscopic information not only at the photoemission threshold, but also using core level and even valence photoelectrons [7]. This becomes possible with XPEEM instruments fitted with energy-filtering systems [8-10]. Measuring in a reliable and reproducible way the performances of XPEEM instruments regarding lateral resolution becomes especially crucial if chemical imaging of nano-objects with core level electrons

\footnotetext{
${ }^{4}$ Present address: CEA-LITEN/DTS/GRETH-LETH, France.
}

is addressed: indeed one needs to know, on the one hand, what will be the smallest distinguishable observable structure, and, on the other hand, what will be the smallest size of two (or more) resolvable adjacent particles. However, the number of experimental parameters influencing directly the result of the measurement drastically complicates this task if the optimal resolution of the instrument needs to be reached. For this reason the theoretical resolution of PEEM instruments is in practice never reached due to the experimental conditions. Authors often state and rely on theoretical figures obtained from electron-optical calculations and which do not reflect 'real life' conditions, which are governed by instrumental (illumination, transmission) and experimental factors regarding the quality of the sample considered. If the factors limiting the resolution are known, their relative impact is rarely quantified. Moreover, reliable measurements of the practical lateral resolution of XPEEM instruments are rather scarce, for two reasons: the first, historical one is linked to the debate among scientists about how lateral resolution should 
be actually measured; this has been clarified quite recently by the International Organization for Standardization within the ISO standards 18 115:2001 (Vocabulary) and 18 516:2006 (Determination of lateral resolution) [11]. The second reason explaining the small number of reliable measurements is the critical lack of an appropriate sample with all the desired surface properties necessary for a consistent XPEEM experiment. To date there is still no agreed solution on how this issue should be addressed, especially for chemical imaging. One of the key questions for reliable measurements that would, for instance, allow for inter-laboratory comparisons, is notably the conditions of the sample preparation (in situ or ex situ), the reproducibility and confidence in the size measurements of the patterns of interest. All of these factors bring in lots of confusion and may be detrimental to a broader use of a now mature XPEEM technique by scientists who, from their readings on the subject, would be willing to apply the technique to new interesting cases.

In this paper, the practical lateral resolution of an energyfiltered XPEEM instrument imaging core level electrons is measured using a cross-sectioned epitaxial multilayer semiconducting sample with certified test patterns offering spatially well defined and chemically sharp interfaces. The sample also enables the use of a simple mathematical model for describing the convolution of the ideal chemical profile with the instrumental response function representing the lateral resolution. A clear difference is made between lateral resolution and spatial sensitivity (i.e., smallest distinguishable object), and the different experimental factors influencing the results (aberrations of electron optics, sample surface imperfections, counting statistics) are presented and quantified. We conclude that the most limiting factor for obtaining high resolutions with core level electrons is the counting statistics, mostly determined by the photon flux inside the microscope field of view.

\section{Experimental details}

\subsection{Sample and method used to determine the lateral resolution}

According to the International Standard ISO documents [11], the lateral resolution of a full-field imaging instrument with an assumed Gaussian response function is defined as the distance between the points at which the signal measured has changed by $12 \%$ of the total signal change in the curve and where the change is $88 \%$ of the total. The lateral resolution then corresponds to the full width at half-maximum (fwhm) of the Gaussian instrumental response function, and is practically measured by a line scan over a straight edge providing a sufficiently sharp chemical gradient; the resulting curve should have a suitable signal as the ordinate and distance as the abscissa. The analysis of gratings [12] is an interesting alternative method to that of using such straight edges, since it is suitable for real time adjustments of XPEEM instruments. Resolution from gratings is estimated from the Rayleigh criterion: if the intensity drop between the intensity maxima $I_{\max }$ of two features separated by $T$ is above $15.2 \%$, then the resolution is $\leqslant 3 / 4 T$. In our experiment, we used a sample enabling resolution measurements from straight edges and gratings, and measurements on isolated features for estimating the size of the smallest detectable object. The sample is an embedded cross-section of epitaxially grown layers of $\mathrm{Al}_{0.7} \mathrm{Ga}_{0.3} \mathrm{As}$ and GaAs on a GaAs substrate [13]. The final mounting of the GaAs platelet has been specifically studied to match the instrumental constraints in order to provide an optimized positioning of the sample surface with respect to the objective lens of the XPEEM. The platelet was thus embedded in a stainless steel disc of $10.8 \mathrm{~mm}$ diameter and $1.6 \mathrm{~mm}$ height and its mounting aligns the surface normal with the optical axis, at $1.8 \mathrm{~mm}$ from the extractor of the spectromicroscope. The surface of the sample provides an array of patterns with strip widths ranging from 0.3 to $700 \mathrm{~nm}$. A schematic drawing of the sample is given in figure 1(a). The combination of square-wave gratings (period $\mathrm{P}_{i}$ from 2 to $600 \mathrm{~nm}$ ), isolated narrow strips (width $\mathrm{W}_{j}$ ) and sharp edges of wide strips offers the possibility of estimating the lateral resolution following various means [12]. As expected for a certified sample [14], a low dispersion (less than 5\%) in the final expected values of $\mathrm{P}_{i}$ and $\mathrm{W}_{j}$ is found by transmission electron microscopy (TEM) or scanning electron microscopy (SEM).

Given the presence of square-wave gratings of known calibrated period, a method of global fitting over the whole image has to be adopted (figure 1(b)) for measuring the instrumental response function. This is also because working with such a limited number of gratings using the Rayleigh criterion gives only a lower and an upper limit for the resolution. An ideal chemical profile of the sample surface was constructed knowing the calibrated distances. The experimental profile determined over the whole field of view and averaged over 200 lines is then considered to be the convolution of the ideal chemical profile and a Gaussian function, the fwhm of which represents the lateral resolution. Mathematically, the convolution of several top-hat functions $\Pi_{a i}$ (width $a_{i}$, centre $x_{i}$ ) with a Gaussian is expressed by

$$
f(x)=\left[\sum_{i} \Pi_{a i}\left(x-x_{i}\right)\right] \otimes \mathrm{e}^{-x^{2} / 2 \sigma^{2}}
$$

which can be expressed using error functions by the following relation:

$$
\begin{aligned}
f(x) & =\sqrt{\frac{\pi}{2}} \sum_{i}\left[\operatorname{erf}\left(\frac{x-x_{i}+a_{i} / 2}{\sqrt{2} \sigma}\right)\right. \\
& \left.-\operatorname{erf}\left(\frac{x-x i-a_{i} / 2}{\sqrt{2} \sigma}\right)\right]
\end{aligned}
$$

where the resolution (Gaussian fwhm) equals $2.35 \sigma$. The fit is performed by a least-squares method using the ideal sample surface chemical profile as the main input.

\subsection{XPEEM imaging conditions}

The XPEEM instrument used here is the NanoESCA spectromicroscope (Omicron Nanotechnology) currently installed at CEA-LETI on the MINATEC Nanocharacterization Centre (Grenoble, France). A detailed description of the principle and 


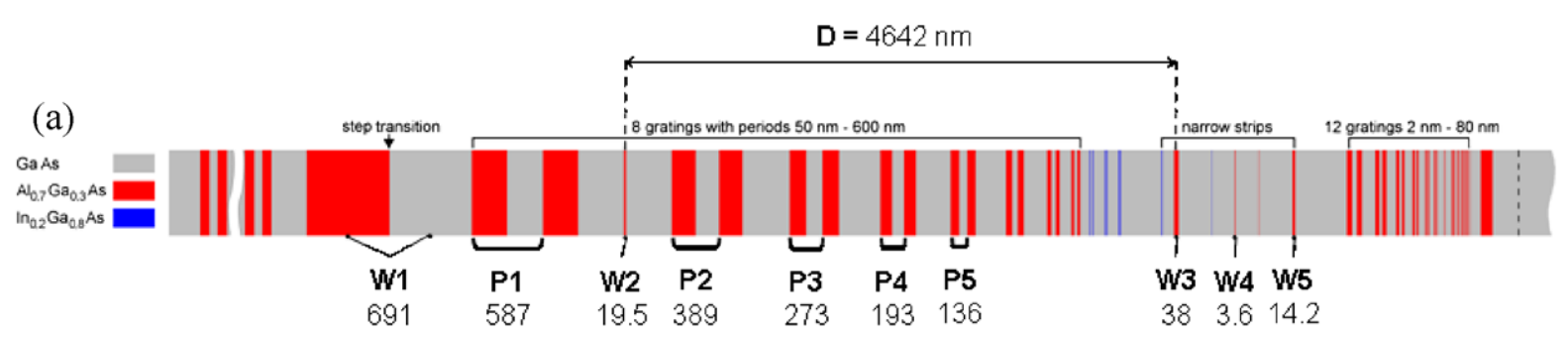

(b)
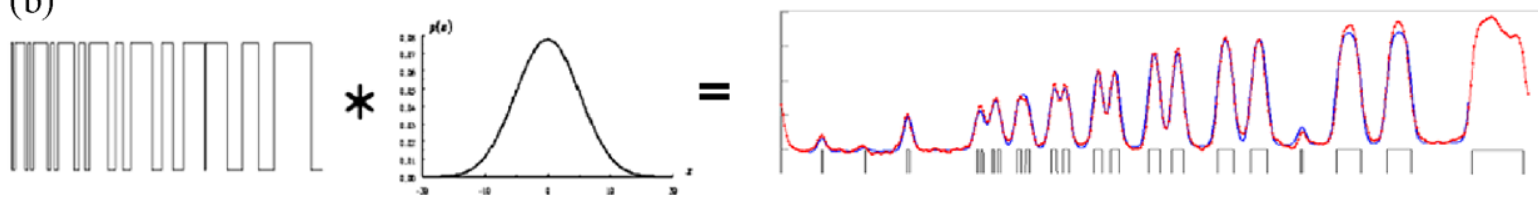

Figure 1. (a) Schematic drawing of the BAM-L200 sample consisting of epitaxially grown layers of $\mathrm{Al}_{0.70} \mathrm{Ga}_{0.30} \mathrm{As}$ (red) and GaAs (light grey) on a GaAs substrate. The surface consists of square-wave gratings (period $\mathrm{P}_{i}$ ) and isolated strips (width $\mathrm{W}_{j}$ ). The distances of interest given in nanometres are certified values obtained by TEM, except $D$ (SEM measurement). The strips labelled W2 to W5 are made of $\mathrm{Al}_{0.70} \mathrm{Ga}_{0.30}$ As. (b) Schematic description of the method used to evaluate the instrument response function (fwhm) from the ideal chemical profile of the sample as shown in (a). The convolution of this profile with a Gaussian instrument response function describes the experimental profile. The fit performed using the least-squares method allows the determination of the fwhm of the Gaussian considered to be equivalent to the lateral resolution.

operation modes of the spectromicroscope can be found elsewhere [8]. It consists of a fully electrostatic PEEM column coupled to a double hemispherical analyser that acts as a high transmission energy filter through correction of the $\alpha^{2}$ aberrations of the electron trajectories occurring in a single analyser. This allows the use of wide, $\mathrm{mm}$ size, entrance slits for imaging at high energy resolution without detrimental effect on the lateral resolution. High lateral resolution is achieved by a combination of an immersion objective lens operated with up to $8.8 \mathrm{kV} \mathrm{mm}^{-1}$ extraction field and a variable size contrast aperture (30-1500 $\mu \mathrm{m})$. The kinetic energy and emission angle spreads of the photoelectrons cause a blurring of the image due to the lens aberrations (chromatic and spherical) which can be reduced by the use of a contrast aperture in the focal back-plane of the objective lens. The instrument used here is normally operated with laboratory excitation sources [9], but for the purpose of this experiment was moved to the European Synchrotron Radiation Facility (ESRF) to benefit from the high brilliance and the energy tuning capabilities of the synchrotron light. The instrument was thus installed on the ID08 soft x-ray beamline delivering photons in the energy range 400-1500 eV. The incident flux was $9.5 \times 10^{12} \mathrm{ph} \mathrm{s}^{-1} \mathrm{~mm}^{-2}(0.1 \% \mathrm{BW})^{-1}$. The beamline parameters were chosen in order to make a compromise between incident intensity at the sample surface, energy resolution and elemental photoionization cross-sections. The incident photon energy was set to $400 \mathrm{eV}$, providing a cross-section for $\mathrm{Ga} 3 \mathrm{~d}$ electrons of $0.8 \mathrm{Mbarn}$; to ensure reasonable statistics, the overall energy resolution was set to $0.9 \mathrm{eV}$, typically achieved with $2 \mathrm{~mm}$ analyser entrance slits and $0.4 \mathrm{eV}$ photon band width. The extractor voltage was $12 \mathrm{kV}$ and the contrast aperture $70 \mu \mathrm{m}$. The field of view, calibrated thanks to the known certified value $D$ of $4642 \pm 24 \mathrm{~nm}$ (see figure 1), was $15.25 \mu \mathrm{m}$. It represents the centre-to-centre distance between the stripes W2 and W3 of 19.5 and $38 \mathrm{~nm}$, respectively. The images were amplified by a multi-channelplate, illuminated a fluorescent screen and were recorded by a twostage Peltier-cooled CCD camera.
Prior to XPEEM imaging, the sample was sputtered in several steps (argon ions of 600-1000 eV) to provide a surface free of contaminants as seen from the XPS survey spectra. The sputtering had only a minor impact on the roughness at interfaces, as checked by atomic force microscopy after the experiment; this topographic effect is discussed in detail below. An additional, reliable way to check for the surface cleanliness is to measure the local work function of each individual layer from an energy-filtered image series at the photoemission threshold [15]. In figure 2 we show two such images at electron energies close to the respective photoemission threshold of $\mathrm{GaAs}$ and $\mathrm{AlGaAs}$, and presenting a clear contrast inversion due to the work function change between the two materials. In the inset are the corresponding reconstructed threshold spectra, from which work functions of $5.1 \mathrm{eV}$ for $\mathrm{GaAs}$ and $5.6 \mathrm{eV}$ for $\mathrm{AlGaAs}$ are extracted. These values are in good agreement with already reported data obtained using Kelvin probe force microscopy (KFM) [16] and show the efficiency of the surface preparation protocol, given the extreme sensitivity of the work function to the chemical cleanliness of surfaces.

\section{Results and discussion}

Figure 3 is the core level image recorded at the maximum intensity of the $\mathrm{Ga} 3 \mathrm{~d}$ core level peak, i.e. at $380.4 \mathrm{eV}$ kinetic energy as determined from the XPS survey spectrum averaged over the field of view. In order to improve the statistics for lateral resolution measurements, a set of 29 images of $15 \mathrm{~min}$ each was acquired and summed, and the dark noise of the CCD camera was subtracted.

It is worth noting that obtaining such a good contrast was not evident a priori since the difference, between two adjacent layers, in gallium atomic concentration is only $35 \%$. From the image we see that the gratings with $587 \mathrm{~nm}$ and the $389 \mathrm{~nm}$ periods (P1 and P2, respectively) are clearly resolved. However for the grating with the $273 \mathrm{~nm}$ period (P3), each 

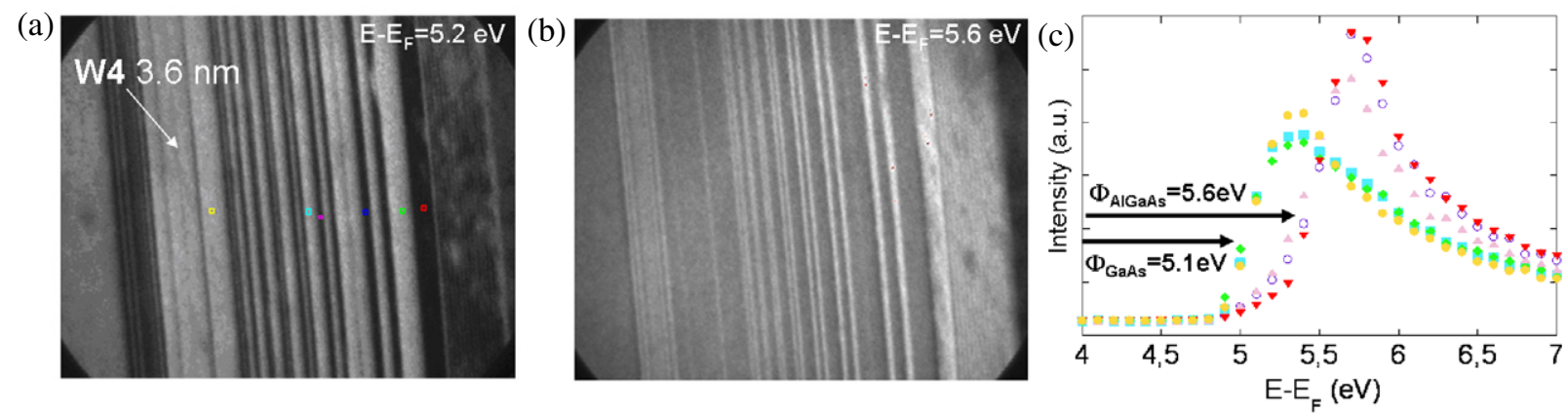

Figure 2. Energy-filtered images taken above the photoemission threshold of (a) $\mathrm{GaAs}\left(E-E_{\mathrm{F}}=5.2 \mathrm{eV}\right.$ ) and (b) $\mathrm{Al}_{0.7} \mathrm{Ga}_{0.3} \mathrm{As}$ $\left(E-E_{\mathrm{F}}=5.6 \mathrm{eV}\right)$ with an excitation energy of $400 \mathrm{eV}$. The field of view is $18 \mu \mathrm{m}$. In image (a) dark zones are AlGaAs layers and bright zones, GaAs layers; in image (b) the contrast is inverted. (c) Extracted threshold nanospectra from the areas marked in (a), from which the characteristic work functions can be derived from fitting of the corresponding local photothreshold. Image (a) shows strip W4 (3.6 nm thick) detectable at near vanishing contrast.

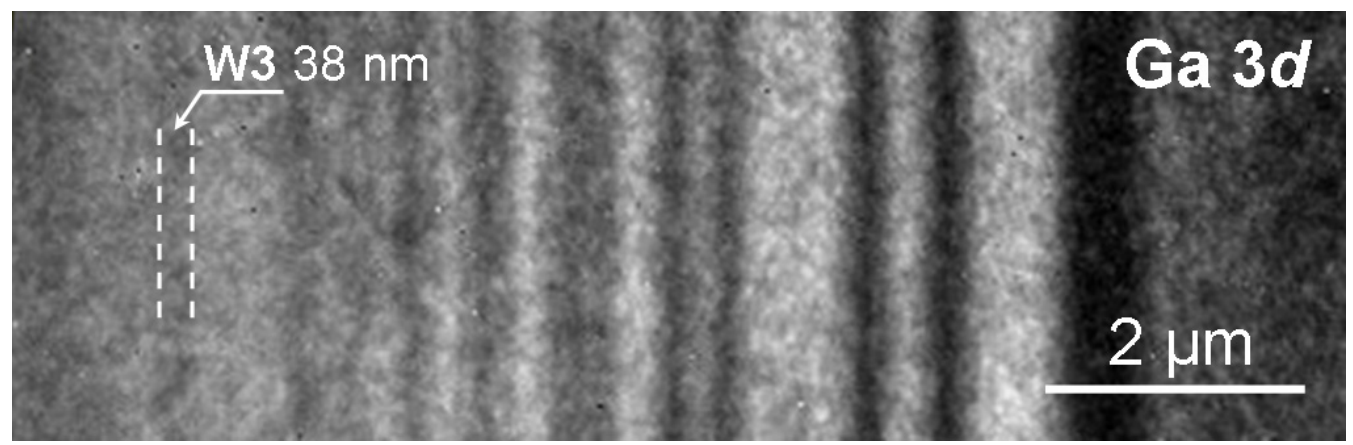

Figure 3. Sum of 29 images ( $15 \mathrm{~min}$ each) acquired at the maximum intensity of the Ga $3 \mathrm{~d}$ core level peak (kinetic energy of $380.4 \mathrm{eV}$; incident $\mathrm{x}$-ray beam of $400 \mathrm{eV})$.

adjacent strip is only faintly distinguishable. The $38 \mathrm{~nm}$ width line (W3) can be identified with a contrast of $\sim 16 \%$.

The intensity profile averaged over 200 lines (i.e., roughly the height of the image figure 3 ) is shown in figure 4 . We see that the Rayleigh criterion is effectively not satisfied for the P3 grating. According to this criterion one can estimate the lateral resolution as being between $3 / 4 \mathrm{P} 2$ and 3/4 P3, i.e. 292 and $205 \mathrm{~nm}$. Following the previously described procedure for fitting the experimental intensity profile, the instrumental response function (i.e. the effective, practical lateral resolution) is equal to $250 \pm 20 \mathrm{~nm}$. The resolution measurement from a step edge as determined on the boundary between the two $700 \mathrm{~nm}$ strips yields $263 \mathrm{~nm}$ and the resolution on the thinnest detectable line of $38 \mathrm{~nm}$ is equal to $270 \mathrm{~nm}$. These three values are in good agreement, indicating a lateral resolution of $\sim 250 \mathrm{~nm}$ for core level imaging with the given experimental conditions. We can first compare this value to a previously published result using the same instrument but under somewhat different conditions. For $\mathrm{Al} 2 \mathrm{p}$ core level images, a resolution of $242 \mathrm{~nm}$ was found with a contrast aperture of $30 \mu \mathrm{m}$ [17], under more favourable illumination conditions (better cross-section) with problems of mechanical stability limiting the acquisition time. In view of the chemical contrast in this latter case $(100 \%)$ being much better than the contrast obtained here with Ga $3 \mathrm{~d}$ photoelectrons (35\%), the result of lateral resolution from the image in figure 3 is therefore equivalent to that previously obtained. However, if we consider the experimental conditions regarding some of the key parameters determining the lateral resolution of the PEEM, and assuming all other parameters optimized (including the focus voltage), i.e. extractor voltage $(12 \mathrm{kV})$ and diameter of the contrast aperture $(70 \mu \mathrm{m})$, the result is far below the theoretical expectations of, roughly, $70 \mathrm{~nm}$. Thus, other factors must play a more important role.

In the following, we recall the different parameters influencing the lateral resolution and estimate their relative impacts in the case of the present experiment. The factors can be classified into three categories, depending on whether they refer to the electron microscope, to the sample itself or to the counting statistics. They are summarized in table 1. Although somewhat arbitrary in the sense that for a cathode lens system, the sample surface is an intrinsic part of the electron optics, the classification above is convenient for clarity in the following discussion.

First, as regards electron-optical factors, the ultimate limit of lateral resolution is given, for a perfect electron lens system, by the diffraction disc of the electrons which depend on the size of the contrast aperture and is smaller than $10 \mathrm{~nm}$ for the diameters used. More important are the chromatic and spherical aberrations of the extractor field (in cathode lens systems), giving theoretical limits in the 10-20 nm range; however attaining these figures requires near perfect alignment 


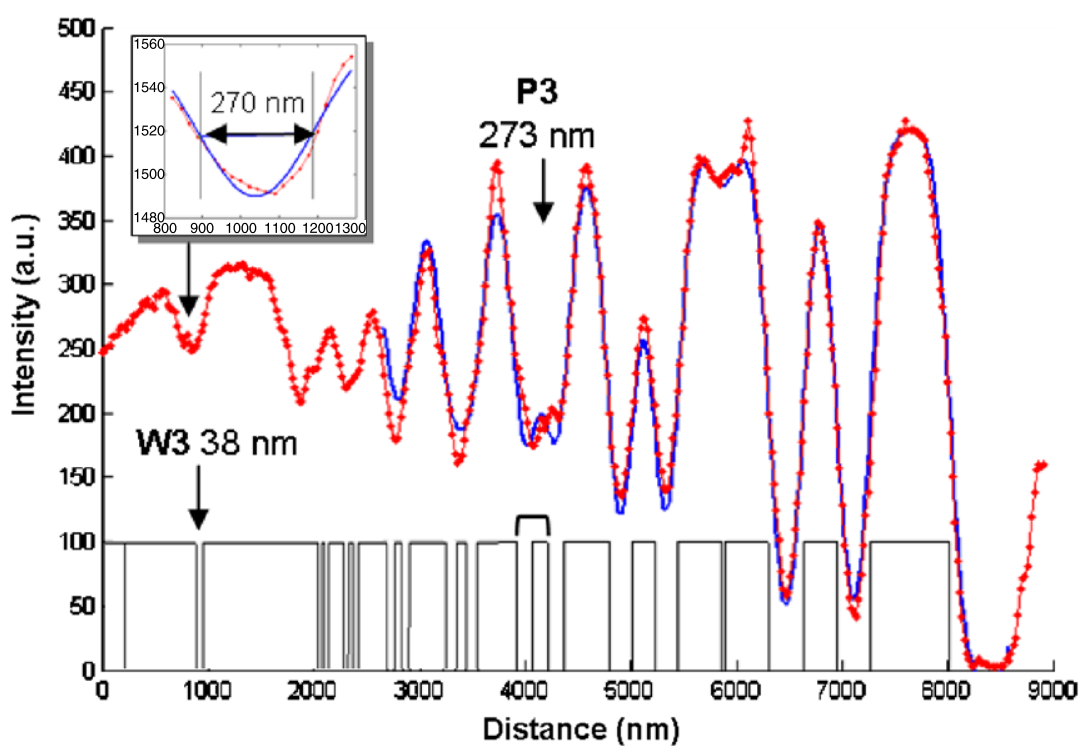

Figure 4. Experimental (dots) and simulated (full line) intensity profile taken across the Ga $3 \mathrm{~d}$ core level image of figure 3 and averaged over 200 rows. The ideal intensity profile used for the fit is displayed at the bottom.

Table 1. Summary of the parameters of interest influencing the lateral resolution in a practical XPEEM experiment.

\begin{tabular}{|c|c|c|}
\hline Source & Experimental parameter & Effect \\
\hline \multirow[t]{5}{*}{ Electron optics } & Extractor accelerating voltage & $\begin{array}{l}\text { Extracting field chromatic and } \\
\text { spherical aberrations }\end{array}$ \\
\hline & Contrast aperture diameter & Decrease of spherical aberrations \\
\hline & Energy filter window & Decrease of chromatic aberrations \\
\hline & Optics mechanical misalignment & Astigmatism \\
\hline & Photoelectron (PE) start angle spread & Extractor/lens spherical aberrations \\
\hline \multirow[t]{3}{*}{ Sample } & Topography & Change of PE start angle spread \\
\hline & Chemical contrast & Contrast ratio \\
\hline & Interface sharpness & Transition width \\
\hline \multirow[t]{3}{*}{ Counting statistics } & Photon flux & Photoemitted intensity \\
\hline & Excitation energy & $\begin{array}{l}\text { PE kinetic energy distribution } \\
\text { and cross-section }\end{array}$ \\
\hline & Microscope transmission & Transmitted intensity \\
\hline
\end{tabular}

and stability of the electron optics. Slight mechanical misalignments within the optics leading to astigmatism can be compensated using an electrostatic octopole (stigmator) provided that suitable (i.e. circular) patterns are present in the field of view. Practically, the best achievable spatial resolution is governed by spherical and chromatic aberrations of the objective lens [1]. Spherical aberrations can be reduced by placing a contrast aperture (several tens of $\mu \mathrm{m}$ ) in the focal back-plane of the objective lens in order to suppress the contribution of electrons that have larger angles to the optical axis, whereas chromatic aberrations are reduced with smaller energy windows set by the energy filter (both of course at the expense of electron flux). In any case a compromise between instrument resolution and image intensity has to be found, keeping in mind that the diameter of the contrast aperture must be as small as possible. The spherical and chromatic aberrations in the electrostatic PEEM column are approximately the sum of those due to the accelerating field and the focusing of the first lens [18]:

$$
\begin{gathered}
d r_{\mathrm{s}} \approx \frac{1}{4}\left(l \frac{E_{0}}{E_{\mathrm{a}}}+C_{\mathrm{s}, \text { lens }}\left(\frac{E_{0}}{E_{\mathrm{a}}}\right)^{3 / 2}\right) \alpha_{\mathrm{o}}^{3} \\
d r_{\mathrm{c}} \approx\left(l \frac{E_{0}}{E_{\mathrm{a}}}+C_{\mathrm{c}, \text { lens }}\left(\frac{E_{0}}{E_{\mathrm{a}}}\right)^{3 / 2}\right) \frac{\Delta E}{E_{0}} \alpha_{\mathrm{o}} .
\end{gathered}
$$

Using our experimental settings $\left(E_{0}=380.4 \mathrm{eV}, E_{\mathrm{a}}=12 \mathrm{kV}\right.$, $\Delta E=1 \mathrm{eV}, 70 \mu \mathrm{m}$ contrast aperture yielding $\alpha \approx 0.87^{\circ}$ for $380 \mathrm{eV}$ electrons) and the appropriate $C_{\mathrm{s}}$ and $C_{\mathrm{c}}$ values of 300 and $130 \mathrm{~mm}$, respectively, this leads to aberrations of $32 \mathrm{~nm}$ in the core level image.

The second set of parameters playing a role in the lateral resolution refers to the properties of the sample, which is an intrinsic component of the cathode objective lens of the PEEM optics. The sample surface is a crucial element of the electron optics since its roughness as well as potential microfields will distort the normally uniform strong acceleration field existing 
between the anode and the sample. A microfield at the sample surface can occur either at sharp topographic defects such as steps, or upon abrupt variations of the work function across the transition being measured. Both are present in our case at each GaAs/AlGaAs interface. Ex situ AFM performed after the experiment indicated a step height difference of 5$8 \mathrm{~nm}$ between the two kinds of layers, the AlGaAs layers protruding with respect to GaAs ones (it was also shown that atomic scale topography was present within each layer). These effects are believed to be mostly due to surface modifications subsequent to ion sputtering (preferential sputtering of GaAs, formation of $\mathrm{Ga}$ droplets [19]); however an initial, though much lower topography, but still of the order of 1-2 nm, due to the preferential oxidation of aluminium, is not to be excluded. As quantitatively shown by Nepijko et al [20], the surface roughness in the form of steps can significantly degrade the measured width of features and hence the lateral resolution which may be deduced. Following the equation for an ideal step with a height of a few nm [20], the deterioration in the lateral resolution is $450 \mathrm{~nm}$ without contrast aperture (in this case the angular image is $4623 \mu \mathrm{m}$ in size), but with a contrast aperture of $70 \mu \mathrm{m}$ limiting the maximum angle of transmitted photoelectrons to $1^{\circ}$, this yields a deterioration of $8 \mathrm{~nm}$. Perturbations of the electric field at the surface can also be the result of a work function step, which has been measured in our experiment (see figure 2(c)) to be $0.5 \mathrm{eV}$ and is equivalent to a step height of $75 \mathrm{~nm}$ in a cathode field of $\sim 6.6 \mathrm{kV} \mathrm{mm}^{-1}$, i.e. about one order of magnitude higher than the electrostatic perturbation arising from a topographic step. Consequently, the deterioration due to surface imperfections is of the order of $80 \mathrm{~nm}$. Combining the two previous estimates of the influence of aberrations $(32 \mathrm{~nm})$ and surface defects $(80 \mathrm{~nm})$, we see that there is still a factor of 3 between the theoretical aberration-limited resolution corrected for sample imperfections (topography-induced and work function-induced microfields) and the experimentally measured value in the core level image of figure 3.

Finally, we consider the influence of the counting statistics on the lateral resolution in core level XPEEM. The photoelectric intensity emitted by electrons of energy $E_{\mathrm{k}}$ and collected from a surface excited by photons of energy $E$ is $I_{\left(E, E_{\mathrm{k}}\right)} \propto \phi_{E} N \sigma_{E} \lambda_{\left(E_{\mathrm{k}}\right)} T_{\left(E_{\mathrm{k}}\right)}, \phi$ being the photon flux at the incident photon energy $E, N$ the number of emitting atoms in the sampled volume, $\sigma$ the cross-section for the core level transition considered, $\lambda$ the photoelectron electron mean free path and $T$ the transmission of the microscope. $T$ is proportional to the square of the radius of the contrast aperture and inversely proportional to $E_{\mathrm{k}}$ in the PEEM column and to $E_{\mathrm{p}}^{2}$ in the energy filter ( $E_{\mathrm{p}}$ being the pass energy). Therefore it plays an important role when core level XPEEM is addressed with $E_{\mathrm{k}}$ values of the order of tens or a few hundreds of $\mathrm{eV}$. In our particular case, with $E_{\mathrm{k}}=380.4 \mathrm{eV}$ and a contrast aperture of $70 \mu \mathrm{m}$ diameter, the microscope transmission was $\sim 4 \times 10^{-4}$. This figure could be one order of magnitude higher at starting energies below $100 \mathrm{eV}$. The incident flux was $9.5 \times 10^{12} \mathrm{ph} \mathrm{s}^{-1} \mathrm{~mm}^{-2}(0.1 \% \mathrm{BW})^{-1}$, equivalent to $\sim 1.7 \times$ $10^{9} \mathrm{ph} \mathrm{s}^{-1}$ within the microscope field of view, a rather low value compared to those for most XPEEM experiments.
Similarly, the cross-section of Ga $3 \mathrm{~d}$ emission could not be fully maximized in the experiment and is $0.8 \mathrm{Mbarn}$ at $400 \mathrm{eV}$ photon energy. Therefore, the overall photoemitted $\mathrm{Ga} 3 \mathrm{~d}$ intensity is low and impacts the count rate, and may play an important role in the observed difference between theoretical and observed lateral resolution.

To account for these conditions, we can introduce a quality factor $Q$ in the form $Q=T^{2} / \delta^{2}$, as originally proposed by Bauer [2], where $T$ is the transmission and $\delta$ the resolution. In its general form, $Q=T^{n} / \delta^{2}$, with the magnitude of the $n$ exponent being a measure for the relative importance of intensity versus resolution. In our particular, 'low intensity' case, we take $n=2$, but have to include the lens part of the aberrations which is omitted in the original calculation [2] and which is the dominant term in our conditions. The generalized quality factor $Q_{2}$ is expressed as

$$
Q_{2}\left(E_{0}, \Delta E, d\right)=\frac{\Delta E^{2} \sin ^{4} \alpha_{0}}{D^{2}}
$$

where $d$ is the diameter of the contrast aperture, $E_{0}$ the starting energy, $\Delta E$ and $\alpha$ the energy and angular spread, and $D$ the diameter of the disc of least confusion resulting from the microscope aberrations. For core level electrons with energies of some $100 \mathrm{eV}$, the quality factor is optimized for a contrast aperture of approximately $350 \mu \mathrm{m}$ diameter according to our calculations. The calculated lateral resolution for this contrast aperture size is limited to about $250 \mathrm{~nm}$, but the transmission rises by a factor of 25 compared to the situation of the present experiment. Thus, the image acquisition time for optimum quality would be reduced to several minutes for comparable signal intensities. In contrast to the long exposure time of more than $7 \mathrm{~h}$ in our experiment, a faster image acquisition helps with operating the instrument in thermal and mechanical stability during one measurement. A combination of optimal focusing (photon beam fwhm: 2-3 times the field of view), brilliance and optimized photoionization cross-sections could gain of two orders of magnitude. This would also reduce data acquisition time, minimizing the risk of mechanical drift, and allowing more precise electron-optical settings to be obtained. In any case the best compromise between incident photon flux and photoionization cross-sections has to be found.

As regards the sample, the use of certified, top-hat-shaped patterns appears extremely useful as the uncertainty in the size is minimized and as it allows inter-laboratory comparisons, provided surface preparation are optimized. It is shown here that with such a sample the effect of nm size topography arising from careful surface preparation has a negligible influence on the effective lateral resolution. Therefore, the use of such an $e x$ situ made sample seems appropriate, provided one can reduce to a minimum the work function step between two consecutive layers with still a good chemical contrast.

Finally, it is important to consider how the determination of the lateral resolution obtained on such a sample having a given number of interface defects (work function step and topography) can be transferred to an unknown sample under study. If we assume similar illumination conditions and microscope settings (particularly the size of the contrast aperture), such a comparison will require us to characterize 
thoroughly the surface topography and work function step at the interface considered in the unknown sample. This means that an ex situ structural characterization using for instance atomic force microscopy will have to be performed in order to determine the height and lateral spread of the features of interest. The topographic data can then be used as input values to determine the lateral resolution deterioration following the mathematical treatment described above.

\section{Conclusion}

We have proposed a method for increasing the reliability of the measurement of the practical lateral resolution of core level XPEEM images and have critically discussed the influence of different experimental factors on the result. The method is based on the use of certified patterns made of crosssectioned epitaxial semiconducting layers (BAM L-200) with low preparation-induced topography $(5-8 \mathrm{~nm}$ ) and on the fitting of the corresponding intensity profile to extract the Gaussian instrumental response function which measures the lateral resolution. With Ga 3d photoelectrons displaying 35\% chemical contrast, and low synchrotron illumination intensity, the measured lateral resolution is $250 \pm 20 \mathrm{~nm}$ and the smallest detectable object is $38 \mathrm{~nm}$. The difference between the experimental results and the aberration-limited theoretical resolution corrected for sample imperfections (topographyinduced and work function-induced microfields) is due to the counting statistics, mainly determined by the photon flux within the field of view. Therefore the combined optimization of sample composition, and excitation (photon energy and flux) seems to be crucial for attaining practical resolutions in XPEEM chemical imaging below $100 \mathrm{~nm}$ on a routine basis.

\section{Acknowledgments}

$\mathrm{AB}$ is grateful to the French ANR programme PNANO 'XPEEM' (ANR 05-NANO-065) for financial support. The authors wish to express their thanks to J C Cezar and G Retout for their skilled help during the beamtime and also to the ESRF review committee for the allowance of beamtime. M Senoner
(BAM) is also gratefully acknowledged for providing useful information regarding the certified sample.

\section{References}

[1] Rempfer G F and Griffith O H 1989 Ultramicroscopy 27273

[2] Bauer E 1991 Ultramicroscopy 3652

[3] Schmidt T et al 2002 Surf. Rev. Lett. 9223

[4] Locatelli A and Bauer E 2008 J. Phys.: Condens. Matter 20093002

[5] Heun S, Watanabe Y, Ressel B, Bottomley D, Schmidt Th and Prince K C 2001 Phys. Rev. B 63125335

Biasiol G, Heun S, Golinelli G B, Locatelli A, Mentes T O, Guo F Z, Hofer C, Teichert C and Sorba L 2005 Appl. Phys. Lett. 87223106

[6] Bailly A, Renault O, Barrett N, Zagonel L F, Gentile P, Pauc N, Dhalluin F, Baron T, Chabli A, Cezar J C and Brookes N B 2008 Nano Lett. 83709

[7] Barrett N, Zagonel L F, Renault O and Bailly A 2009 J. Phys.: Condens. Matter 21314015

[8] Escher E, Weber N, Merkel M, Ziethen C, Bernhard P, Schönhense G, Schmidt S, Forster F, Reinert F, Krömeker B and Funnemann D 2005 J. Phys.: Condens. Matter 14 S1329

[9] Renault O, Barrett N, Bailly A, Zagonel L F, Mariolle D, Cezar J C, Brookes N B, Winkler K, Krömeker B and Funnemann D 2007 Surf. Sci. 6014727

[10] Locatelli A, Bianco A and Cocco D 2003 J. Physique IV 10499

[11] Seah M P 2001 Surf. Interface Anal. 311048 Wolsentholme J 2008 Surf. Interface Anal. 40966

[12] Wirth T, Senoner M, Unger W, Osterle W, Kaiander I, Sellin R L and Bimberg D 2005 Nanoscale Calibration Standards and Methods ed G Wilkening and L Koenders (Weinheim: Wiley-VCH) p 282

[13] Senoner M, Wirth T and Unger W 2004 Surf. Interface Anal. 361423

[14] Unger W and Senoner M www.bam.de/en/fachthemen/ referenzmaterialen/index.html

[15] Renault O, Brochier R, Roule A and Haumesser P H 2006 Surf. Interface Anal. $\mathbf{3 8} 375$

[16] Usunami T, Mizutani T, Kishimoto S and Maezawa K 1999 Japan. J. Appl. Phys. 38 L767

[17] Senoner M, Wirth T, Unger W, Escher M, Weber N, Funnemann D and Krömeker B 2005 J. Surf. Anal. 1278

[18] Watts R N, Liang S and Levine Z H 1997 Rev. Sci. Instrum. 683464

[19] Senoner M 2007 private communication

[20] Nepijko S A, Sedov N N, Schönhense G, Escher M, Bao X and Huang W 2000 Ann. Phys., Lpz. 6441 